\title{
SNR 1987A: the Birth of a Supernova Remnant
}

\author{
Richard McCray \\ JILA, University of Colorado, Boulder, CO 80309-0440, USA
}

\begin{abstract}
In 1996, the blast wave from Supernova 1987A began to strike the inner circumstellar ring, causing the appearance of "hot spots" on the ring. This event marks the birth of the supernova remnant, SNR1987A, defined as the epoch when the light of the event is dominated by interaction of the expanding debris of the supernova with its circumstellar matter.
\end{abstract}

\section{Introduction}

Today, we are observing SN1987A approximately 15 years after its initial outburst. During the first 10 years, the radiation from SN1987A was dominated by energy deposited in the interior by the decay of newly synthesized radioisotopes. From observations of this radiation at many wavelengths, we learned a great deal about the dynamics and thermodynamics of the expanding debris. With the Hubble Space Telescope, we have also observed a remarkable system of three circumstellar rings, the origin of which remains a mystery today.

About 6 years ago, the blast wave from the supernova began to strike the inner circumstellar ring, resulting in the appearance of a rapidly brightening "hot spot" on the ring. Today, many more hot spots have appeared, and the radio, infrared, optical, and X-ray radiation from of the supernova is now dominated by the impact of the supernova debris with its circumstellar matter. This impact marks the birth of a supernova remnant, SNR1987A.

Here, I discuss what we know about the circumstellar matter and rings, and what we are learning from observations of the interaction of the supernova debris with the circumstellar matter. Finally, I will hazard a few guesses about what we can expect to learn from SNR1987A during the next few decades.

\section{Energetics}

First, it might be useful to summarize the main energy sources of SN1987A. These are listed in Table 1.

As Table 1 shows, SN1987A has three different sources of energy, each of which emerges as a different kind of radiation and with a different timescale. The greatest is the collapse energy itself, which emerges as a neutrino burst lasting a few seconds. The energy provided by radioactive decay of newly synthesized elements is primarily responsible for the optical display. Most of this energy emerged within the first year after outburst, primarily in optical and infrared 
emission lines and continuum from relatively cool $(T \lesssim 5,000 \mathrm{~K})$ gas. Note that the radioactive energy is relatively small, $\sim 10^{-4}$ of the collapse energy.

Table 1: SN1987A Energetics

\begin{tabular}{|c|c|c|c|}
\hline Source & Collapse & Radioactivity & Expansion \\
\hline Definition & $\sim \frac{G M_{\odot}^{2}}{R_{N^{*}}}$ & $\begin{array}{c}{ }^{56} \mathrm{Ni} \rightarrow^{56} \mathrm{Co} \rightarrow \rightarrow^{56} \mathrm{Fe} \\
\left(0.07 M_{\odot}\right)\end{array}$ & $\int_{\text {debris }} \frac{1}{2} V$ \\
\hline Emerges as: & $\begin{array}{l}\text { Neutrinos } \\
(k T \sim 4 M e V)\end{array}$ & $\begin{array}{l}\mathrm{O}, \mathrm{IR} \\
(+\mathrm{X}, \gamma)\end{array}$ & $\begin{array}{l}\text { X-rays } \\
(+\mathrm{R}, \mathrm{IR}, \mathrm{O}, \mathrm{UV})\end{array}$ \\
\hline Energy [ergs] & $10^{53}$ & $10^{49}$ & $10^{51}$ \\
\hline Timescale & $\sim 10$ seconds & $\sim 1$ year & $\sim 10-1000$ years \\
\hline
\end{tabular}

The kinetic energy of the expanding debris will be converted into radiation when the supernova debris strikes circumstellar matter. When this happens, two shocks always develop: the blast wave, which overtakes the circumstellar matter; and the reverse shock, which is driven inwards (in a Lagrangean sense) through the expanding debris. The gas trapped between these two shocks is typically raised to temperatures in the range $10^{6}-10^{8} \mathrm{~K}$ and will radiate most of its thermal energy as X-rays with a spectrum dominated by emission lines in the range $0.3-10 \mathrm{keV}$.

Most of the kinetic energy of the debris will not be converted into thermal energy of shocked gas until the blast wave has overtaken a circumstellar mass comparable to that of the debris itself, $\sim 10-20 \mathrm{M}_{\odot}$. Typically, that takes many centuries, and as a result, most galactic supernova remnants (e.g., Cas A) reach their peak X-ray luminosities after a few centuries and fade thereafter.

As I shall describe, we believe that SN1987A is surrounded by a few $\mathrm{M}_{\odot}$ of circumstellar matter within a distance of parsec or two. Thus, a significant fraction of the kinetic energy of the debris will be converted into thermal energy within a few decades as the supernova blast wave overtakes this matter.

\section{The Circumstellar Rings}

The first evidence for circumstellar matter around SN1987A appeared a few months after outburst in the form of narrow optical and ultraviolet emission lines seen with the International Ultraviolet Explorer (Fransson et al. 1993) Even before astronomers could image this matter, they could infer that:

- the gas was nearly stationary (from the linewidths);

- it was probably ejected by the supernova progenitor (because the abundance of nitrogen was elevated);

- it was ionized by soft X-rays from the supernova flash (from emission lines of NV and other highly ionized elements in the spectrum);

- it was located at a distance of about a light year from the supernova (from the rise time of the light curve of these lines); and

- the gas had atomic density $\sim 3 \times 10^{3}-3 \times 10^{4} \mathrm{~cm}^{-3}$ (from the fading timescale of the narrow lines - Lundqvist \& Fransson 1996). 
Figure 1 shows an image of the circumstellar rings of SN1987A taken with the WFPC on the Hubble Space Telescope. Dividing the radius of the inner ring (0.67 lt-year) by the radial expansion velocity of the inner $\operatorname{ring}\left(\approx 10 \mathrm{~km} \mathrm{~s}^{-1}\right.$ Crotts \& Heathcote 1991 ) gives a kinematic timescale $\approx 20,000$ years since the gas in the ring was ejected, assuming constant velocity expansion. The more distant outer loops are expanding more rapidly, consistent with the notion that they were ejected at the same time as the inner ring.

The rings observed by $H S T$ may be only the tip of the iceberg. They are glowing by virtue of the ionization and heating caused by the flash of EUV and soft X-rays emitted by the supernova during the first few hours after outburst. But calculations (Ensman \& Burrows 1992) show that this flash was a feeble one. The glowing gas that we see in the triple ring system is probably only the ionized inner skin of a much greater mass of unseen gas that the supernova flash failed to ionize. For example, the inner ring has a glowing mass of only about $\sim 0.04 \mathrm{M}_{\odot}$, just about what one would expect such a flash to produce.

In fact, ground-based observations of optical light echoes during the first few years after outburst provided clear evidence of a much greater mass of circumstellar gas within several light years of the supernova that did not become ionized (Crotts et al. 1989; Wang \& Wampler 1992). The echoes were caused by scattering of the optical light from the supernova by dust grains in this gas. They became invisible a few years after outburst.

What accounts for this circumstellar matter and the morphology of the rings? I suspect that the supernova progenitor was originally a close binary system, and that the two stars merged some 20,000 years ago. The inner ring might be the inner rim of a circumstellar disk that was expelled during the merger, perhaps as a stream of gas that spiraled out from the outer Lagrangean (L2) point of the binary system. Then, during the subsequent 20,000 years before the supernova event, ionizing photons and stellar wind from the merged blue giant star eroded a huge hole in the disk. Finally, the supernova flash ionized the inner rim of the disk, creating the inner ring that we see today.

The binary hypothesis provides a natural explanation of the bipolar symmetry of the system, and may also explain why the progenitor of SN1987A was a blue giant rather than a red giant (Podsiadlowski 1992). But we still lack a satisfactory explanation for the outer loops. If we could only see the invisible circumstellar matter that lies beyond the loops, we might have a chance of reconstructing the mass ejection episode.

Fortunately, SN1987A will give us another chance. When the supernova blast wave hits the inner ring, the ensuing radiation will cast a new light on the circumstellar matter. As I describe below, this event is now underway.

\section{The Crash Begins}

The first evidence that the supernova debris was beginning to interact with circumstellar matter came from radio and X-ray observations. As Figure 2 shows, SN1987A became a detectable source of radio and soft X-ray emission about 1200 days after the explosion and has been brightening steadily in both bands ever since. Shortly afterwards, astronomers imaged the radio source with the Australia Telescope Compact Array $(A T C A)$ and found that the radio source 


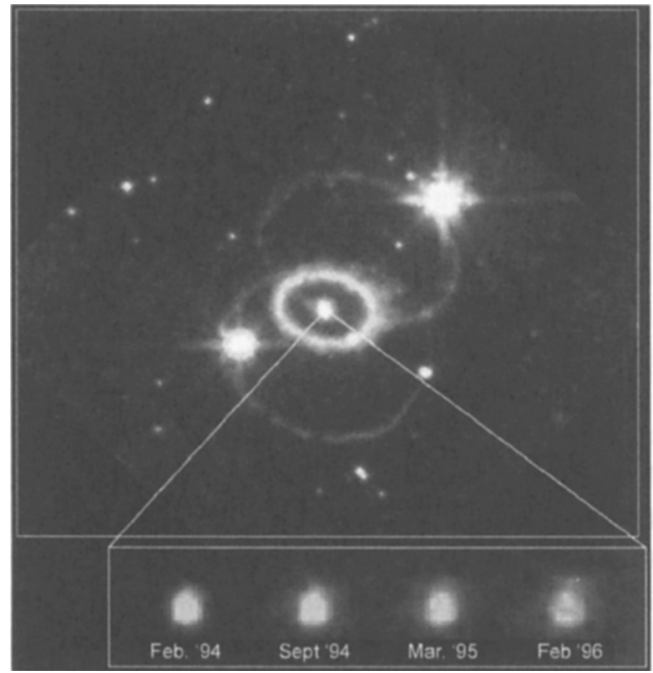

Figure 1. HST image of SN1987A and its circumstellar rings. The inset at the bottom shows the evolution of the glowing center of the supernova debris.

was an elliptical annulus inside the inner circumstellar ring observed by $H S T$ (Figure 7). From subsequent observations, they found that the annulus was expanding with a velocity $\sim 3,500 \mathrm{~km} \mathrm{~s}^{-1}$ (Gaensler et al. 2000).

The radio emission most likely comes from relativistic electrons accelerated by shocks formed inside the inner ring where the supernova debris struck relatively low density $\left(n \sim 100 \mathrm{~cm}^{-3}\right)$ circumstellar matter, and the X-ray emission comes from the shocked circumstellar matter and supernova debris (Chevalier 1992). Subsequently, Chevalier \& Dwarkadas (1995) suggested a model for the circumstellar matter, in which the inner circumstellar ring is the waist of an hourglass-shaped bipolar nebula. The low-density circumstellar matter is a thick layer of photoionized gas that lines the interior of the bipolar nebula. The inner boundary of this layer is determined by balance of the pressure of the hot bubble of shocked stellar wind gas and that of the photoionized layer. In the equatorial plane, the inner boundary of this layer is located at about half the radius of the inner ring. According to this model, the appearance of X-ray and radio emission at $\sim 1200$ days marks the time when the blast wave first enters the photoionized layer.

\section{The Reverse Shock}

Borkowski et al. (1997a) used a 2-D hydro code to simulate the impact of the outer atmosphere of the supernova with an idealized model for the photoionized layer. They found a good fit to the ROSAT observations with a model in which the thickness of the photoionized layer was about half the radius of the inner ring and the layer had atomic density $n_{0} \approx 150 \mathrm{~cm}^{-3}$. 

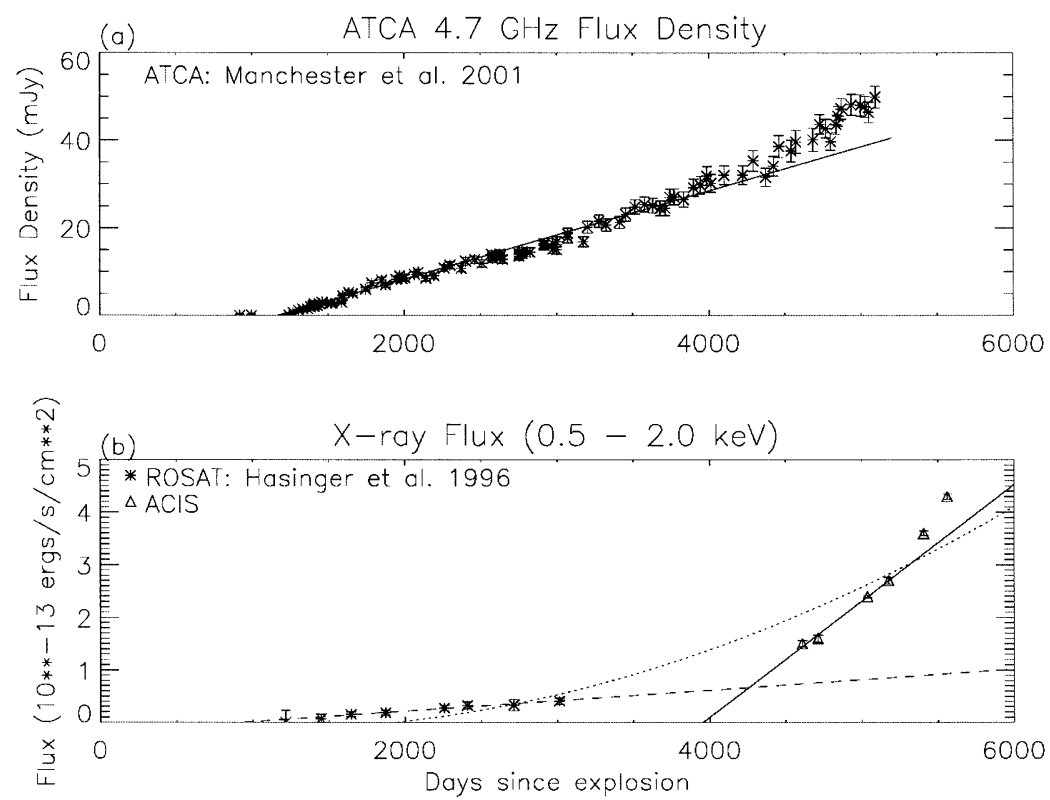

Figure 2. Radio (upper) and X-ray (lower) light curves.

The same model predicted that $\mathrm{L} \alpha$ and $\mathrm{H} \alpha$ emitted by hydrogen atoms crossing the reverse shock should be detectable with the STIS. Then, in May 1997 , only three months after these predictions were published, the first STIS observations of SN1987A were made, and broad $\left(\Delta V \approx \pm 12,000 \mathrm{~km} \mathrm{~s}^{-1}\right) \mathrm{L} \alpha$ emission lines were detected (Sonneborn et al. 1998). Within the observational uncertainties, the flux was exactly as predicted (Michael et al 1998).

The broad $\mathrm{L} \alpha$ and $\mathrm{H} \alpha$ emission lines are not produced by recombination. (The emission measure of the shocked gas is far too low to produce detectable L $\alpha$ and $\mathrm{H} \alpha$ by recombination.) Instead, the lines are produced by neutral hydrogen atoms in the supernova debris as they cross the reverse shock and are excited by collisions with electrons and protons in the shocked gas. Since the cross sections for excitation of the $n \gtrsim 2$ levels of hydrogen are nearly equal to the cross sections for impact ionization, about one $\mathrm{L} \alpha$ photon is produced for each hydrogen atom that crosses the shock. Thus, the observed flux of broad $\mathrm{L} \alpha$ is a direct measure of the flux of hydrogen atoms that cross the shock. Moreover, since the outer supernova envelope is expected to be nearly neutral, the observed flux is a measure of the mass flux across the shock.

The fact that the $\mathrm{L} \alpha$ and $\mathrm{H} \alpha$ lines are produced by excitation at the reverse shock gives us a powerful tool to map this shock. Since any hydrogen in the supernova debris is freely expanding, its line-of-sight velocity, $V_{\|}=z / t$, where $z$ is its depth measured from the mid-plane of the debris and $t$ is the time since the supernova explosion. Therefore, the Doppler shift of the L $\alpha$ line will be directly proportional to the depth of the reverse shock: $\Delta \lambda / \lambda_{0}=z / c t$. Thus, by mapping the $\mathrm{L} \alpha$ or $\mathrm{H} \alpha$ emission with STIS, we can generate a 3-dimensional image of the reverse shock. 


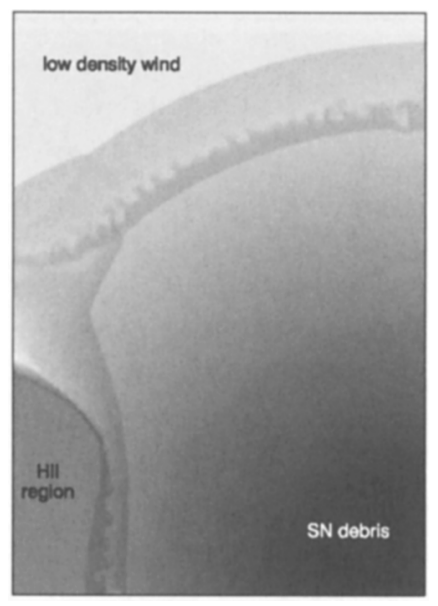

Figure 3. Hydrodynamic simulation of the interaction of SN1987A debris with circumstellar matter (Michael et al. 2003). The supernova center is at the lower right corner and the gray scale indicates density. The figure is cylindrically symmetric about the right boundary, and the photoionized layer is assumed to be a thick torus (labeled HII region). A blast wave enters the photoionized layer (HII region), while a reverse shock arrests the free expansion of the supernova debris. Between the blast wave and the reverse shock are layers of shocked HII region and shocked supernova debris, separated by an unstable contact discontinuity.

Figure 4 illustrates this procedure. Panel a shows the location of the slit superposed on an image of the inner circumstellar ring, with the near $(\mathbf{N})$ side of the tilted ring on the lower left. Panel $\mathbf{b}$ shows the actual STIS spectrum of $\mathrm{L} \alpha$ from this observation. The slit is black due to geocoronal L $\alpha$ emission. The bright blue-shifted streak of L $\alpha$ extending to the left of the lower end of the slit comes from hydrogen atoms crossing the near side of the reverse shock, while the fainter red-shifted streak at the upper end of the slit comes from the far side of the reverse shock.

From this and similar observations with other slit locations we have constructed a map of the reverse shock surface, shown in panel c. Note that the emitting surface is an annulus that lies inside the inner circumstellar ring, as would be expected from a hydrodynamic model such as that illustrated in Figure 3 . Presumably, the reverse shock in the polar directions lies at a greater distance from the supernova, where the flux of atoms in the supernova debris is too low to produce detectable emission. Panel $\mathbf{d}$ is a model of the STIS L $\alpha$ spectrum that would be expected from hydrogen atoms crossing the shock surface illustrated in panel c. By comparing such model spectra with the actual spectra (e.g., panel b), we may refine our model of the shock surface (Michael et al. 2003).

Note that the broad L $\alpha$ emission is much brighter on the near (blue-shifted) side of the debris than on the far side, and so is the reconstructed shock surface. There is one obvious reason why this might be so: the blue-shifted side of the 
reverse shock is nearer to us by several light-months, and so we see the emission from the near side as it was several months later than that from the far side. Since the flux of atoms across the reverse shock is increasing, the near side should be brighter. But this explanation fails quantitatively. The observed asymmetry is several times greater than can be explained by light-travel time delays. Moreover, the asymmetry is much greater in $\mathrm{L} \alpha$ than it is in $\mathrm{H} \alpha$. Recently, Michael et al. (2003) have proposed that most of the asymmetry in $\mathrm{L} \alpha$ can be attributed to resonant scattering of $\mathrm{L} \alpha$ in the un-shocked supernova debris, which tends to reflect the $\mathrm{L} \alpha$ emission outward. However, the $\mathrm{H} \alpha$ emission line from the reverse shock is not susceptible to resonant scattering, so the $(\sim 30 \%)$ asymmetry in $\mathrm{H} \alpha$ and must be attributed in part to real asymmetry in the supernova debris. As we shall see, observations at radio and X-ray wavelengths also provide compelling evidence for asymmetry of the supernova debris.

\section{The Hot Spots}

In April 1997, Sonneborn et al. (1998) obtained the first STIS spectrum of SN1987A with the $2 \times 2$ arcsecond aperture. Images of the circumstellar ring were seen in several optical emission lines. No Doppler velocity spreading was evident in the ring images except at one point, located at $P . A .=29^{\circ}(\mathrm{E}$ of $\mathrm{N})$, which we now call "Spot 1," where a Doppler-broadened streak was seen in $\mathrm{H} \alpha$ and other optical lines.

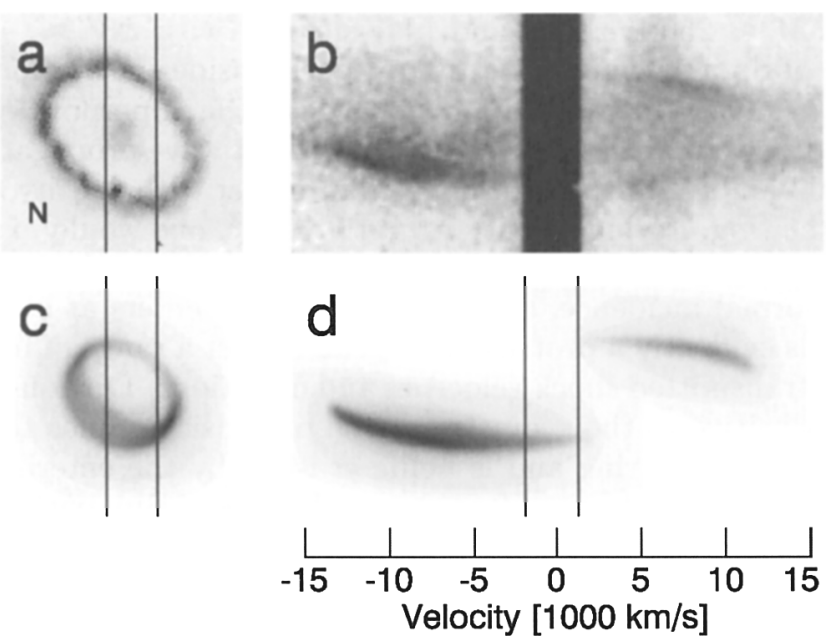

Figure 4. STIS spectrum of $\mathrm{L} \alpha$ emission from the reverse shock (Michael et al. 1998)

Figure 5 (Pun et al. 2002) shows a portion of a more recent (March 1998) STIS spectrum of Spot 1, where one can see vertical pairs of bright spots corresponding to emission from the stationary ring at $\mathrm{H} \alpha$ and [NII] $\lambda \lambda 6548,6584$. (One also sees three more fainter spots at each wavelength where the outer 

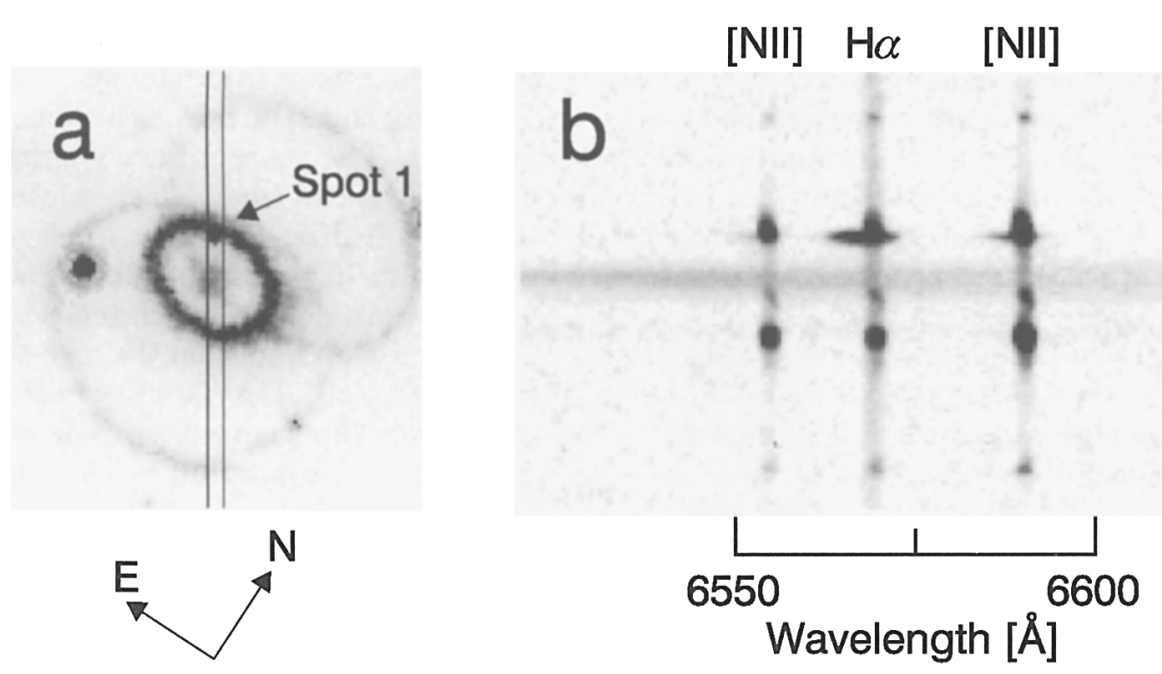

Figure 5. Spectrum of Spot 1. (a) Slit orientation on image of SN1987A's triple ring system; (b) Section of STIS G750M spectrum.

loops cross the slit, and a broad horizontal streak at the center due to the $\mathrm{H} \alpha$ emission from the rapidly expanding inner debris. The broad $\mathrm{H} \alpha$ from the reverse shock is invisible at this dispersion.) The emission lines are broadened (with $F W H M \approx 250 \mathrm{~km} \mathrm{~s}^{-1}$ ) and blue-shifted (with $\Delta V \approx-80 \mathrm{~km} \mathrm{~s}^{-1}$ ) at the location of Spot 1, which is located slightly inside the stationary ring.

Spot 1 evidently marks the location where the supernova blast wave first touches the dense circumstellar ring. When a blast wave propagating with velocity $V_{b} \approx 4,000 \mathrm{~km} \mathrm{~s}^{-1}$ through circumstellar matter with density $n_{0} \approx 150 \mathrm{~cm}^{-3}$ encounters the ring, having density $n_{r} \approx 10^{4} \mathrm{~cm}^{-3}$, one would expect the transmitted shock to propagate into the ring with $V_{r} \approx\left(n_{0} / n_{r}\right)^{1 / 2} V_{b} \approx 500 \mathrm{~km} \mathrm{~s}^{-1}$ if it enters at normal incidence, and more slowly if it enters at oblique incidence. Since Spot 1 is evidently a protrusion, we can expect a range of incidence angles, and hence of transmitted shock velocities and directions. Obviously, the line profiles will be sensitive to the geometry of the protrusion. Since the protrusion is on the near side of the ring and is being crushed by the entering shocks, most of the emission will be blue-shifted, as is observed. But part of the emission is red-shifted because it comes from oblique shocks entering the far side of the protrusion.

Spot 2 did not appear until November 1998, but shortly thereafter, several more spots appeared (Sugerman et al. 2002). By May 2002, 16 spots were evident. The hot spots are brightening rapidly, with doubling timescales ranging from a few months to 2 years.

The emission line spectrum of Spot 1 resembles that of a radiative shock, in which the shocked gas has had time to cool from its post-shock temperature $T_{1} \approx 1.6 \times 10^{5}\left[V_{r} /\left(100 \mathrm{~km} \mathrm{~s}^{-1}\right)\right]^{2} \mathrm{~K}$ to a final temperature $T_{f} \approx 10^{4} \mathrm{~K}$ or less. As the shocked gas cools, it is compressed by a density ratio $n_{f} / n_{r} \approx$ $\left(T_{1} / T_{f}\right) \approx 160\left[V_{r} /\left(100 \mathrm{~km} \mathrm{~s}^{-1}\right)\right]^{2}\left[T_{f} /\left(10^{4} \mathrm{~K}\right)\right]^{-1}$. We see evidence of this com- 

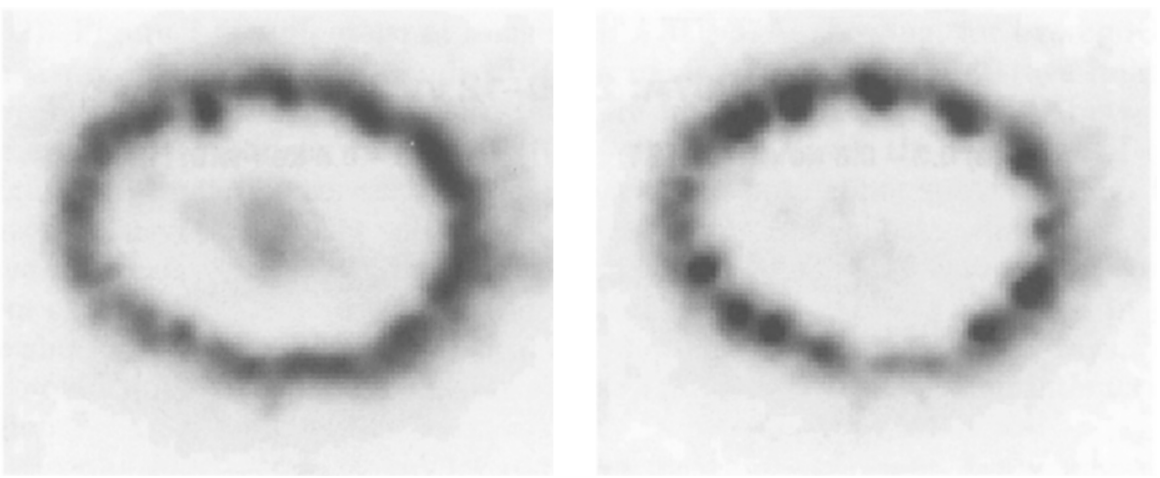

Figure 6. WFPC narrow-band $\mathrm{H} \alpha$ images of the inner ring. Left: February 1996. Right: May 2002. Both the inner ring and the central supernova debris have faded monotonically. In February 1996, only Spot 1, located at P.A. $029^{\circ}$ is visible. By May 2002, 16 hot spots have appeared.

pression in the observed ratios of forbidden lines, such as [NII] $\lambda \lambda 6548,6584$ and $[\mathrm{SII}] \lambda \lambda 6717,6731$, from which we infer electron densities in the range $n_{e} \sim$ $10^{6} \mathrm{~cm}^{-3}$ using standard nebular diagnostics (Pun et al. 2002).

The fact that the shocked gas in Spot 1 was able to cool and form a radiative layer within a few years sets a lower limit, $n_{r} \gtrsim 10^{4} \mathrm{~cm}^{-3}$, on the density of unshocked gas in the protrusion. Given that limit, we can estimate an upper limit on the emitting surface area of Spot 1 , from which we infer that Spot 1 should have an actual size no greater than about one pixel on the HST Wide Field Planetary Camera (WFPC2). This result is consistent with the imaging observations.

The cooling timescale of shocked gas is sensitive to the postshock temperature, hence shock velocity. For $n_{r}=10^{4} \mathrm{~cm}^{-3}$, shocks faster than $250 \mathrm{~km} \mathrm{~s}{ }^{-1}$ will not be able to radiate and form a cooling layer within a few years. It is quite possible that such fast non-radiative shocks are present in the protrusions. For example, I estimated above that a blast wave entering the protrusion at normal incidence might have velocity $\sim 500 \mathrm{~km} \mathrm{~s}^{-1}$. Faster shocks would be invisible in optical and UV line emission, but we are probably seeing evidence of such shocks in soft X-rays $(\S 7)$. We would still see the optical and UV line emission from the slower oblique shocks on the sides of the protrusion, however.

\section{The X-ray Source}

As I have already mentioned in $\S 4$, we believe that the X-ray emission from SNR1987A seen by ROSAT (Figure 2) comes from the hot shocked gas trapped between the supernova blast wave and the reverse shock. But, with its 10 ! angular resolution, ROSAT was unable to image this emission; nor was it able to obtain a spectrum.

Our ability to analyze the X-rays from SNR1987A advanced dramatically with the launch of the Chandra observatory (Burrows et al. 2000; Park et al. 


\section{SNR 1987A: 2000-12 vs 2002-05}

(a) $0.3-0.8 \mathrm{keV}$ with HST

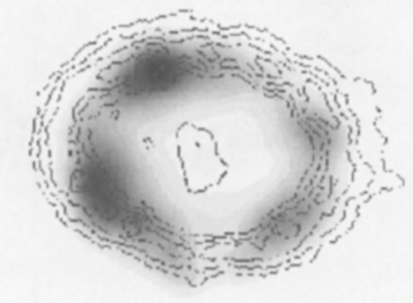

(c) $0.8-1.2 \mathrm{keV}$ with HST

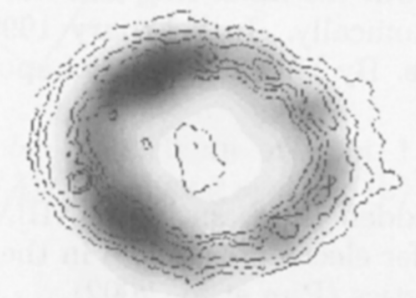

(ө) $1.2-8.0 \mathrm{keV}$ with ATCA (b) $0.3-0.8 \mathrm{keV}$ with HST

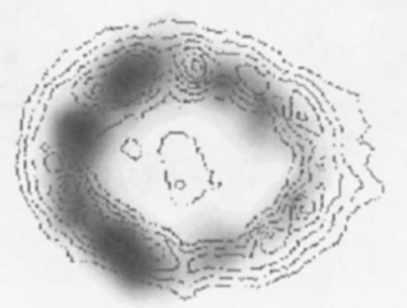

(d) $0.8-1.2 \mathrm{keV}$ with HST

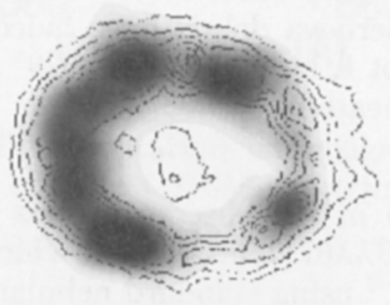

(f) 1.2 - $8.0 \mathrm{keV}$ with ATCA

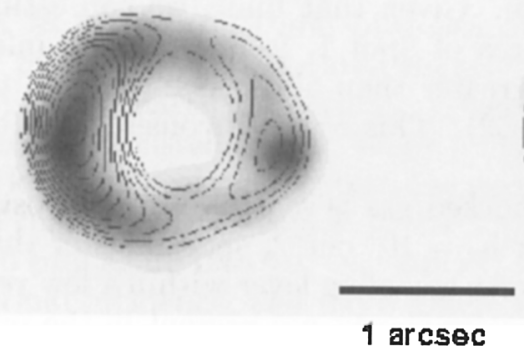

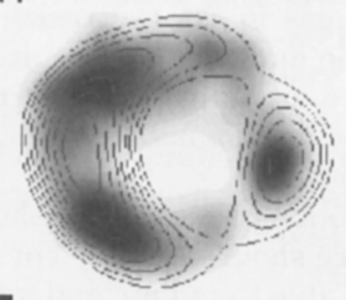

Figure 7. Optical, radio and X-ray images of SNR1987A. North is to the top. The images on the left $(\mathrm{a}, \mathrm{c}, \mathrm{e})$ represent observations of Dec. 2000 , while those on the right $(\mathrm{b}, \mathrm{d}, \mathrm{f})$ represent observations of May 2002. The gray scale images represent the X-ray brightness in three bands. The contour lines in the top four images $(a-d)$ represent the optical ring as seen by HST, while those in the bottom two represent the radio image as seen by ATCA. 
2002). Figure 7 is a montage of images of SNR1987A, showing, for two epochs, the X-ray images in three bands. We see immediately that the X-rays images have brightened and changed substantially in the 18-month interval between these two observations. In the soft $(0.3-0.8 \mathrm{keV})$ band, the locations of the bright X-ray emission correlate fairly well with the optical hot spots ( $c f$. Fig. 6). This fact suggests that the softer $\mathrm{X}$-rays are coming from shocks where the blast wave is entering the inner ring. But we can be sure that the X-rays do not come from exactly the same shocks as the optical hot spots. As we discussed in $\S 6$, the shocks responsible for the optical emission are too slow to emit X-rays, but we would not be surprised if faster shocks are present in the same interaction region.

In the hard $(1.2-8 \mathrm{keV})$ band, the locations of bright X-ray emission correlate better with the images from the $A T C A$ radio array than with the optical hot spots. This fact suggests that the harder X-rays are produced primarily by the hotter gas between the reverse shock and the blast wave (cf. Fig. 3). We presume that the radio emission would correlate with the harder X-rays because the relativistic electrons responsible for the non-thermal radio emission have energy density proportional to that in the X-ray emitting gas and reside in roughly the same volume.

The fact that the X-ray and radio images are both brighter on the $\mathbf{E}$ (left) side than on the $\mathbf{W}$ could be explained by a model in which either: (a) the circumstellar gas inside the inner ring had greater density toward the E; or (b) the outer supernova debris had greater density toward the E. But the fact that most of the hot spots appeared first on the $\mathbf{E}$ side favors the latter hypothesis. If the circumstellar gas had greater density toward the $\mathbf{E}$ side and the supernova debris were symmetric, the blast wave would have propagated further toward the $\mathbf{W}$ side, and the hot spots would have appeared there first.

This conclusion is also supported by observations of $\mathrm{H} \alpha$ and $\mathrm{L} \alpha$ emission from the reverse shock ( $\S 5)$, which show that the flux of mass across the reverse shock is greater on the $\mathbf{W}$ side.

These observations highlight a new puzzle about SN1987A: why was the explosion so asymmetric? We might explain a lack of spherical symmetry by rapid rotation of the progenitor, but how do we explain a lack of azimuthal symmetry?

With the grating spectrometer on Chandra, we have also obtained a spectrum of the X-rays from SNR1987A, shown in Figure 8 (Michael et al. 2002). It is dominated by emission lines from helium- and hydrogen-like ions of $\mathrm{O}, \mathrm{Ne}, \mathrm{Mg}$, and $\mathrm{Si}$, as well as a complex of Fe-L lines near $1 \mathrm{keV}$, as predicted (Borkowski et al 1997b). The characteristic electron temperature inferred from the spectrum, $k T_{e} \sim 3 \mathrm{keV}$, is much less than the proton temperature, $k T_{p} \sim 30 \mathrm{keV}$ for a blast wave propagating with $V_{b} \approx 4,000 \mathrm{~km} \mathrm{~s}^{-1}$ and that inferred from the widths of the X-ray emission lines. This result is a consequence of the fact that Coulomb collisions are too slow to raise the electron temperature to equilibrium with the ions.

The Chandra observations (Fig. 2) show that the current X-ray flux from SNR1987A is more than 3 times the value that would be estimated by extrapolating the ROSAT light curve to December 2001 (Figure 2). The acceleration in the brightening rate suggests that most of the $\mathrm{X}$-rays are now coming from the 


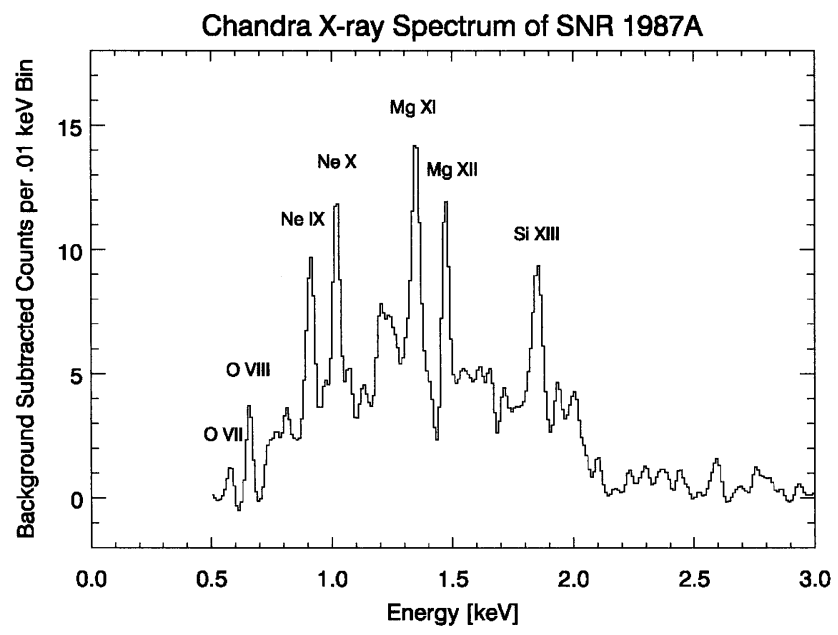

Figure 8. X-ray spectrum of SNR1987A.

impact of the blast wave with the ring. The X-ray flux is expected to increase by another factor $\sim 10^{2}$ during the coming decade as the blast wave overtakes the inner circumstellar ring (Borkowski et al. 1997b).

\section{The Future}

SNR1987A has been tremendous fun so far but the best is yet to come. During the next ten years, the blast wave will overtake the entire circumstellar ring. More hot spots will appear, brighten, and eventually merge until the entire ring is blazing brighter than Spot 1 . We expect that the $\mathrm{H} \alpha$ flux from the entire ring will increase to $F_{\mathrm{H} \alpha} \gtrsim 3 \times 10^{-12} \mathrm{ergs} \mathrm{cm}^{-2} \mathrm{~s}^{-1}$, or $\gtrsim 30$ times brighter than it is today and that the flux of ultraviolet lines will be even greater (Luo et al. 1994).

As we have already begun to see, observations at many wavelength bands are needed to tell the entire story of the birth of SNR1987A. Fortunately, powerful new telescopes and technologies are becoming available just in time to witness this event.

Large ground-based telescopes equipped with adaptive optics will provide excellent optical and infrared spectra of the hot spots. We need to observe profiles of several emission lines at high resolution in order to unravel the complex hydrodynamics of the hot spots. These telescopes also offer the exciting possibility to image the source in infrared coronal lines of highly ionized elements (e.g., [Si IX] 2.58, 3.92 $\mu \mathrm{m},[\mathrm{Si} \mathrm{X}] 1.43 \mu \mathrm{m}$ ) that may be too faint to see with $H S T$. Observations in such lines will complement X-ray observations to measure the physical conditions in the very hot shocked gas.

The observations with the $A T C A$ have given us our first glimpse of shock acceleration of relativistic electrons in real time, but the angular resolution of 
$A T C A$ is not quite good enough to allow a detailed correlation of the radio image with the optical and X-ray images. This correlation will become possible several years from now when the Atacama Large Millimeter Array $(A L M A)$ is completed. Such observations will give us a unique opportunity to test our theories of relativistic particle acceleration by shocks.

Of course, we should continue to map the emission of fast $\mathrm{L} \alpha$ and $\mathrm{H} \alpha$ from the reverse shock with $S T I S$. Such observations give us a three-dimensional image of the flow of the supernova debris across the reverse shock, providing the highest resolution map of the asymmetric supernova debris. We expect this emission to brighten rapidly, doubling on a timescale $\sim 1$ year. Most exciting, such observations will give us an opportunity to map the distribution of nucleosynthesis products in the supernova debris. We know that the debris has a heterogeneous composition. The early emergence of gamma rays from SN1987A showed that some of the newly synthesized ${ }^{56} \mathrm{Co}$ (and probably also clumps of oxygen and other elements) were mixed fairly far out into the supernova envelope by instabilities following the explosion (McCray 1993). When such clumps cross the reverse shock, the fast $\mathrm{H} \alpha$ and $\mathrm{L} \alpha$ lines will vanish at those locations, to be replaced by lines of other elements. If we keep watching with $S T I S$, we should see this happen during the coming decade.

The shocks in the hot spots are surely producing ionizing radiation, roughly half of which will propagate ahead of the shock and ionize heretofore invisible material in the rings. The effects of this precursor ionization will soon become evident in the form of narrow cores in the emission lines from the vicinity of the hot spots.

In $\S 3$ I pointed out that the circumstellar rings of SN1987A represent only the inner skin of a much greater mass of circumstellar matter, and that we obtained only a fleeting glimpse of this matter through ground-based observations of light echoes. The clues to the origin of the circumstellar ring system lie in the distribution and velocity of this matter, if only we could see it clearly. Fortunately, SNR1987A will give us another chance. Although it will take several decades before the blast wave reaches the outer rings, the impact with the inner ring will eventually produce enough ionizing radiation to cause the unseen matter to become an emission nebula. We have estimated (Luo et al 1994) that the fluence of ionizing radiation from the impact will equal the initial ionizing flash of the supernova within a few years after the ring reaches maximum brightness. I expect that the circumstellar nebula of SNR1987A will be in full flower within a decade. In this way, SN1987A will be illuminating its own past.

\section{References}

Borkowski, K. J., Blondin, J. M., \& McCray, R. 1997a, ApJ, 476, L31

Borkowski, K., Blondin, J. M., \& McCray, R. 1997b, ApJ, 477, 281

Burrows, D. N. et al. 2000, ApJ, 543, L149

Chevalier, R. A. 1992, Nature, 355, 617

Chevalier, R. A. \& Dwarkadas, V. I. 1995, ApJ, 452, L45

Crotts, A., \& Heathcote, S. R. 1991, Nature, 350, 683

Crotts, A. P. S., Kunkel, W. E., \& McCarthy, P. J. 1989, ApJ, 347, L61 
Ensman, L. \& Burrows, A. 1992, ApJ, 393, 742

Fransson, C. et al. 1993, ApJ, 336, 429

Gaensler, B. M. et al. 2000, Astr. Soc. Pacific Conf. Ser. 199, 449

Lundqvist, P. \& Fransson, C. 1996, ApJ, 464, 924

Luo, D., McCray, R., \& Slavin, J. 1994, ApJ, 430, 264

McCray, R. 1993, ARA\&A, 31, 175

Michael, E. et al. 1998, ApJ, 492, L139

Michael, E. et al. 2002, ApJ, 574, 166

Michael, E. et al. 2003, to be published (astro-ph )

Park, S. et al. 2002, ApJ, 567, 314

Podsiadlowski, P. 1992, PASP, 104, 717

Pun, C. S. J. et al. 2002, ApJ, 572, 906

Sonneborn, G. et al. 1998, ApJ, 492, L143

Sugerman, B. E. K. et al. 2002, ApJ, 572, 209

Wang, L. \& Wampler, E. J. 1992, A\&A, 262, L9 